\title{
A SHORT PROOF OF THE F. AND M. RIESZ THEOREM
}

BERNT K. ØKSENDAL ${ }^{1}$

In this note we provide a short proof of the following theorem due to F. and M. Riesz:

TheOREM. Let $\mu$ be a complex Borel measure on the unit circle $T$ in the complex plane such that $\int p d \mu=0$ for all polynomials $p$. Then $\mu$ is absolutely continuous with respect to Lebesgue measure on $T$.

Proof. Let $E$ be a Borel set on $T$ with length zero and let $F$ be a compact subset of $E$. It suffices to prove that $\mu(F)=0$. Choose an integer $n$ and cover $F$ by open discs $\Delta_{1}, \cdots, \Delta_{N}$ with centers $z_{1}, \cdots$, $z_{N}$ in $F$ and radii $r_{1}, \cdots, r_{N}$ respectively such that $\sum_{i=1}^{N} r_{i}<1 / n^{2}$. Put $\rho_{i}=n \cdot r_{i}$ and define

$$
g_{n}(z)=1-\prod_{i=1}^{N} \frac{z-z_{i}}{z-\left(1+\rho_{i}\right) z_{i}} .
$$

Then $\left|g_{n}(z)\right| \leqq 2$ for $z \in T$ and $g_{n}$ can be uniformly approximated on $T$ by polynomials, so $\int g_{n} d \mu=0$. If $z \in F$ then $z \in \Delta_{i}$ for some $i$, and hence $\left|g_{n}(z)-1\right| \leqq r_{i} / \rho_{i}=1 / n$. If $z \in T$ and $|z-y| \geqq \delta>0$ for all $y \in F$, then we have

$$
\begin{aligned}
\left|g_{n}(z)\right| & =\left|1-\prod_{i=1}^{N}\left(1+\frac{\rho_{i} z_{i}}{z-\left(1+\rho_{i}\right) z_{i}}\right)\right| \\
& \leqq \prod_{i=1}^{N}\left(1+\frac{\rho_{i}}{\left|z-\left(1+\rho_{i}\right) z_{i}\right|}\right)-1 \\
& \leqq \exp \left(\sum_{i=1}^{N} \log \left(1+\rho_{i} / \delta\right)\right)-1 \leqq \exp \left(\sum_{i=1}^{N} \rho_{i} / \delta\right)-1 \\
& =\exp \left(n \delta^{-1} \sum_{i=1}^{N} r_{i}\right)-1<\exp \left(\frac{1}{n \delta}\right)-1 .
\end{aligned}
$$

So $g_{n}(z) \rightarrow 1$ for $z \in F$ and $g_{n}(z) \rightarrow 0$ for $z \in T \backslash F$ as $n \rightarrow \infty$. By dominated convergence, $\mu(F)=\lim _{n \rightarrow \infty} \int g_{n} d \mu=0$, and the proof is complete.

University of California, Los Angeles, California 90024.

Received by the editors March 22, 1971.

AMS 1970 subject classifications. Primary 41A10; Secondary 28A10.

Key words and phrases. Unit circle, polynomial, Borel measure, F. and M. Riesz Theorem.

1 This work was partially supported by NSF Grant GP-11475 and NAVF, Norway.

Copyright (1) 1971, American Mathematical Society 